\title{
Impact ionization of molecular oxygen by 3.5-MeV/u bare carbon ions
}

\author{
Saikat Nandi, ${ }^{1}$ Aditya N. Agnihotri, ${ }^{1}$ S. Kasthurirangan, ${ }^{2}$ Ajay Kumar, ${ }^{3}$ Carmen A. Tachino, ${ }^{4}$ \\ Roberto D. Rivarola, ${ }^{4}$ F. Martín, ${ }^{5,6}$ and Lokesh C. Tribedi ${ }^{1, *}$ \\ ${ }^{1}$ Tata Institute of Fundamental Research, Homi Bhabha Road, Colaba, Mumbai 400005 , India \\ ${ }^{2}$ Institute of Chemical Technology, Matunga, Mumbai 400 019, India \\ ${ }^{3}$ NPD, Bhabha Atomic Research Center, Mumbai 400 085, India \\ ${ }^{4}$ Institute de Fisica Rosario (CONICET-UNR), Universidad Nacional de Rosario, 2000 Rosario, Argentina \\ ${ }^{5}$ Departamento de Química, Modulo 13, Universidad Autónoma de Madrid, 28049 Madrid, Spain \\ ${ }^{6}$ Instituto Madrileño de Estudios Avanzados en Nanociencia, Cantoblanco, 28049 Madrid, Spain
}

(Received 3 January 2012; revised manuscript received 25 April 2012; published 18 June 2012)

\begin{abstract}
We have measured the absolute double-differential cross sections (DDCSs) for electron emission in ionization of $\mathrm{O}_{2}$ molecules under the impact of 3.5-MeV/u C $\mathrm{C}^{6+}$ ions. The data were collected between 10 and $600 \mathrm{eV}$, in an angular range of $30^{\circ}$ to $150^{\circ}$. The single-differential cross sections (SDCSs) in emission angle and electron energy are deduced from the electron DDCS spectra. Also, the total cross section has been obtained from the SDCS spectra. The DDCS spectra as well as the SDCS spectra are compared with continuum distorted-wave eikonal initial-state calculations which employ molecular wave functions built as linear combinations of atomic orbitals. The DDCS ratio i.e. $\sigma_{\mathrm{O} 2} / 2 \sigma_{\mathrm{O}}$, derived by dividing the experimental DDCS for molecular oxygen with the theoretical DDCS for atomic oxygen, does not show any primary or secondary oscillations arising from Young-type interference, which is apparently in contrast to what has been observed earlier for $\mathrm{H}_{2}$ and in agreement with the model calculation. Similarly, the forward-backward angular asymmetry increases monotonically with the velocity of the emitted electrons. However, the results on the DDCSs, SDCSs, the asymmetry parameter, and the nonexistence of oscillations are in qualitative agreement with the predictions of the model used.
\end{abstract}

DOI: 10.1103/PhysRevA.85.062705

PACS number(s): $34.50 . \mathrm{Gb}$

\section{INTRODUCTION}

The collision of multiply charged heavy ions with diatomic molecules provides a way to study different inelastic processes such as Coulomb ionization, electron capture to the continuum, and electronic excitation of the target molecule. Unlike electrons and lighter ions, such as protons, as projectiles, in heavy-ion collisions the two-center effect plays a major role in the motion of the secondary electrons in the final state of ionization. The two-center mechanism has been found to influence the angular distribution of the low-energy electron emission which has been studied in detail mostly using low- $Z$ (atomic number) targets like $\mathrm{H}_{2}$ and He. These distributions are in large disagreement with the calculations predicted from the first-order Born approximation. Several theoretical [1,2] and experimental [3-7] studies indicate the success of the quantum-mechanical models based on continuum distorted-wave approximations. Double-differential cross section (DDCS) studies of low-energy electrons emitted in ionization of medium- $Z$ atoms and molecules by different projectiles have been reported in a few cases [8-11]. In addition to the interest of studying two-center electron emission mechanisms, recently investigations on molecular ionization have been extended following the observation of Young-type electron interference in ionization of a homonuclear diatomic molecule. It was predicted earlier [12-14] that a diatomic molecule can act as a molecular double slit in ionization by photons, electrons, or heavy ions, which is revealed as oscillations in the electron emission spectrum due to the interference effect. Now there has been rapid progress in this

\footnotetext{
*Electronic address: lokesh@tifr.res.in
}

field through ionization studies of $\mathrm{H}_{2}$ molecules both experimentally [15-22] and theoretically [23,24]. Even the existence of a double-frequency component in the oscillatory structure has been debated [25-27] in the case of ionization of $\mathrm{H}_{2}$.

Such studies are relatively scarce for multielectron systems, except for a few experimental $[10,11]$ works based on $\mathrm{N}_{2}$ and $\mathrm{O}_{2}$ and theoretical calculations [28] on $\mathrm{N}_{2}$ ionization by fast-proton impact. However, the theoretical model [28] does not reveal any interference patterns in the ionization cross section of $\mathrm{N}_{2}$. This behavior was physically explained for $\mathrm{N}_{2}$ targets in that the oscillatory structure in DDCS ratios corresponding to each initial molecular orbital contribution is shifted with respect to the others so that when added no undulations are observed [28]. This prediction, however, is in contrast with the observations by Baran et al. [10]. In the present work, we have used fast $3.5-\mathrm{MeV} / \mathrm{u} \mathrm{C}^{6+}$ ions to collide with $\mathrm{O}_{2}$ molecules. The velocity being quite high ( $v=11.86$ a.u.), the electron capture probability can be quite low and Coulomb ionization is the main mechanism for electron emission. We have investigated the details of the low-energy electron emission via a study of the energy and angular distribution of double-differential cross sections of electron emission. One of the motivations for our work is to look for the presence or absence of the oscillatory structure in the electron emission (with a period of about 2.5 a.u. in the electron velocity [11]), which has been debated in these recent experimental and theoretical works.

Besides studying the interference oscillation, the other motivation of this work is to measure a set of absolute DDCSs over a wide energy and angular range to provide a stringent test for the predictions of the recent continuum distorted-wave (CDW) eikonal intial-state (EIS) model calculations, which 
use suitable molecular wave functions of the $\mathrm{O}_{2}$ molecule developed for this work. We divide the paper into the following sections: an overview of the experiment, description of the theoretical model, results and discussion including the data analysis, and conclusions. The absolute DDCS data are also provided in tabular form for ready reference.

\section{OVERVIEW OF THE EXPERIMENT}

The present experiments were performed with $3.5-\mathrm{MeV} / \mathrm{u}$ $\mathrm{C}^{6+}$ ions available from the 14-MV BARC-TIFR Pelletron accelerator facility at Mumbai, India. The energy- and chargestate-analyzed $\mathrm{C}^{4+}$ ion beam was made to pass through a postaccelerator carbon foil stripper to obtain ions of higher charge states, including bare ions. The bare ions were then selected through the switching magnet system and directed to the specific beamline. The beam was then collimated into the desired size using a pair of four-jawed slits $(2 \times$ $2 \mathrm{~mm}^{2}$ ) mounted $1 \mathrm{~m}$ apart along the beamline. These are followed by another aperture of $4 \mathrm{~mm}$ diameter. This act of collimation resulted in a parallel beam of desired size, which thereby reduced the probability of producing forward-moving electrons due to slit scattering that could contribute to the background counts at extreme forward angles. Finally, the beam was passed through a differential pumping arrangement followed by the interaction chamber. The scattering chamber was flooded with the target gas (molecular oxygen) at pressure about 0.15 mTorr. A capacitance manometer (MKS Baratron) was used to measure the absolute pressure of the target gas inside the chamber. The inner side of the scattering chamber was lined with two sets of thin $\mu$-metal sheets (thickness $0.3 \mathrm{~mm}$ ) in order to reduce earth's magnetic field at about 5-10 mG near the interaction region. The secondary electrons emitted in the collision process were energy analyzed by a hemispherical electrostatic energy analyzer with its inner and outer electrodes having radii 2.5 and $3.5 \mathrm{~cm}$, respectively. The energy-analyzed electrons were finally detected using a channel electron multiplier (CEM) placed at the exit slit of the analyzer. The cone of the CEM was kept at a positive potential of $100 \mathrm{~V}$, so that the electrons incident on the CEM have energies between 100 and $500 \mathrm{eV}$ and, in consequence, the detection efficiency of the CEM remains constant. For each angle, the number of electrons at different energies that were ejected in that direction was detected for a specified amount of incident projectile charge collected on a Faraday cup. The double-differential cross sections were obtained from the measured electron spectrum at a given angle by the electron spectrum taken with and without target and the relation given by Ref. [29]

$$
\frac{d^{2} \sigma}{d \Omega_{e} d \epsilon_{e}}=\frac{\frac{N_{e}\left(\epsilon_{e}, \theta e\right)}{N_{p} \Delta \epsilon}-\frac{N_{b}\left(\epsilon_{e}, \theta e\right)}{N_{p}^{\prime} \Delta \epsilon}}{n(l \Omega)_{\mathrm{eff}} \eta_{\mathrm{el}}},
$$

where $n=\left[9.659 \times 10^{15} P_{c}\right.$ (mTorr) $] /[T(\mathrm{~K})]$ is the number density of the target in a static gas pressure condition. The quantities $N_{e}$ and $N_{b}$ are the number of electrons detected in the presence and absence of the target gas, respectively. The numbers of incident projectile ions are represented as $N_{p}$ and $N_{p}^{\prime}$, in the presence and absence of the target gas, respectively. The quantity $\Delta \epsilon$ is the energy resolution of the spectrometer, which is typically $6 \%$ of the electron energy. The detection efficiency of the CEM, $\eta_{\mathrm{el}}$, is taken to be 0.83 , as mentioned in the operation manual [30] of the CEM used. Finally, $(l \Omega)_{\mathrm{eff}}$ is the solid-angle path-length integral given by $\frac{w_{1} w_{2} h_{2}}{L R \sin \theta}$, where $w_{1}, w_{2}, h_{1}$, and $h_{2}$ are defined as the widths and heights for the entrance and exit slits of the spectrometer, respectively [29,31]. The length of the collimator is $L$ and $R$ is the distance of the collimator from the center of the interaction zone. $(l \Omega)_{\mathrm{eff}}$ is minimum at $90^{\circ}$ and increases for other scattering angles. There could be two different orientation of the spectrometer for which it makes an angle $90^{\circ}$ with respect to the projectile beam. The DDCS spectra obtained at these two positions match well (within 7\%-8\%) with each other. This indicates a good alignment of the collimator of the spectrometer with respect to the direction of the projectile beam. The energy dependence of the DDCSs was studied for ten different angles, i.e., $30^{\circ}$, $45^{\circ}, 60^{\circ}, 75^{\circ}, 80^{\circ}, 90^{\circ}, 105^{\circ}, 120^{\circ}, 135^{\circ}$, and $150^{\circ}$, and at each angle the secondary electrons having energies between 10 and $600 \mathrm{eV}$ were detected. Some of the representative values of absolute electron DDCSs have been tabulated in Table I. The error due to statistical fluctuation was low (5\%-10\%) throughout the experiment. The uncertainty in the gas pressure was about $6 \%-7 \%$. Overall, the maximum absolute error in the data presented here is about $15 \%-18 \%$.

\section{THEORETICAL MODELS}

Details concerning the theoretical model employed to describe single-electron ionization from diatomic or polyatomic molecules have already been given in a previous work [28]; thus, only a general description of the method will be presented in this work. The bound state $\varphi_{i_{\mathrm{MO}}}$ of the active electron in a molecular orbital (MO) is described by employing a basis set of Slater-type orbitals (STOs) $\phi_{h, j}^{\text {sTO }}$ centered on each target nucleus. So, considering a molecular frame of reference whose origin is located at the center of mass of the molecule and with the $z^{\prime}$ axis oriented along the direction of the molecular internuclear axis, the wave function $\varphi_{i_{M O}}$ is written as follows:

$$
\varphi_{i_{\mathrm{MO}}}(\vec{r})=\sum_{h, j} \omega_{h, j} \phi_{h, j}^{\mathrm{STO}}\left(\vec{x}_{h}\right),
$$

where the index $h(h=1,2)$ indicates the molecular nuclei in which the STOs are centered, whereas the index $j$ represents the set of quantum numbers $n l m$. Also, in expression (2), the vectors $\vec{r}$ and $\vec{x}_{h}$ denote the electron coordinates with respect to the center of mass of the molecule and the target nucleus $h$, respectively. To obtain the optimized values of both the STO exponents and the coefficients $\omega_{h, j}$ the quantum chemistry program GAUSSIAN 98 [32] was employed; in particular a minimal STO-6G basis set $[33,34]$ within the unrestricted Hartree-Fock (UHF) approximation was chosen to calculate them. A larger UHF/6-311G*basis set was used to obtain the equilibrium internuclear distance $b$ and the orbital energy $\varepsilon_{i}$ values.

Double-differential cross sections for a molecular orbital as a function of the energy $\varepsilon_{k}$ and the orientation $\Omega_{k}$ of the emitted electron are calculated, in the CDW-EIS model, by integrating the corresponding postform of the scattering matrix element $\mathcal{R}_{i, f_{\text {MO }}}^{+, \text {CDW-EIS }}$ over the transverse momentum transfer $\vec{\eta}$ 


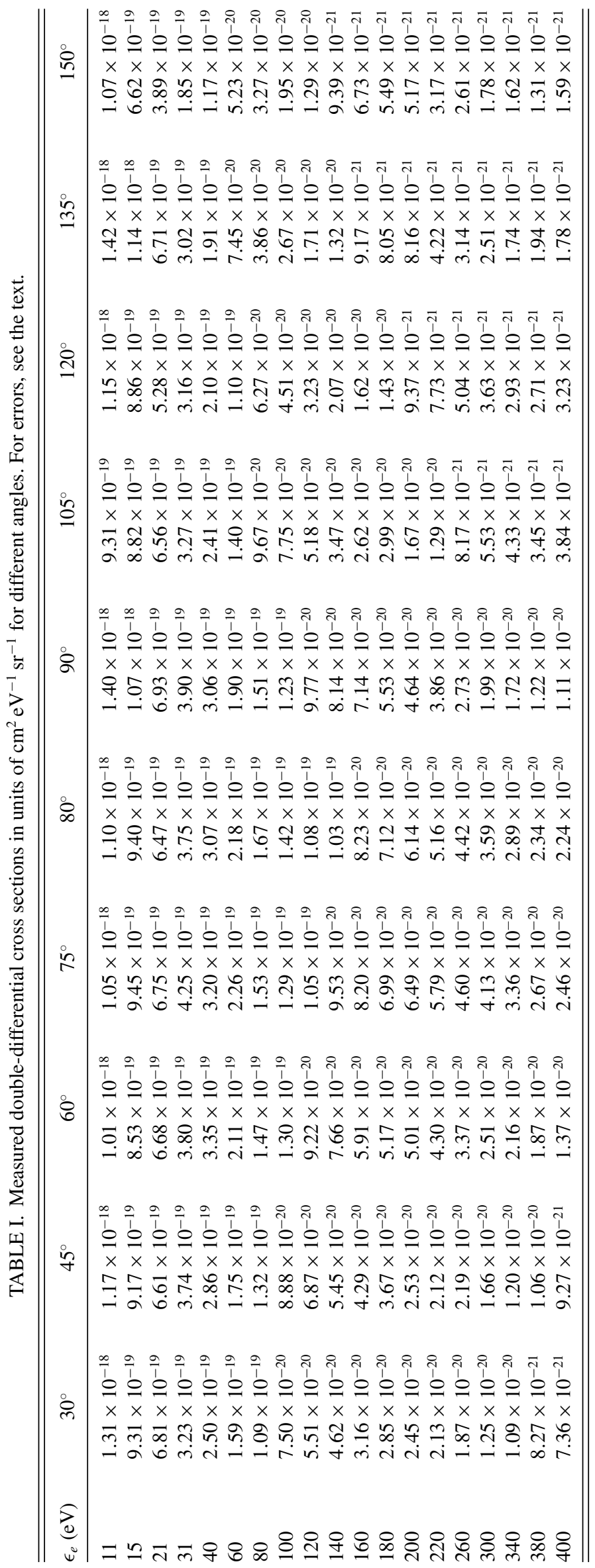


and averaging then over all possible molecular orientations:

$$
\sigma_{\mathrm{MO}}^{(2)}\left(\varepsilon_{k}, \Omega_{k}\right)=\frac{N_{\mathrm{MO}}}{4 \pi} k \iint d \vec{\eta} d \Omega_{b}\left|\mathcal{R}_{i, f_{\mathrm{MO}}}^{+, \text {CDW-EIS }}\left(\vec{\eta}, \Omega_{b}\right)\right|^{2}
$$

with $N_{\mathrm{MO}}$ the occupation number of the molecular orbital, $k=\sqrt{2 \varepsilon_{k}}$ the momentum of the emitted electron, and $\Omega_{b}$ the molecular solid angle. It can be shown that the square modulus of the scattering matrix element $\mathcal{R}_{i, f_{\mathrm{MO}}}^{+, \mathrm{CD}-\mathrm{EIS}}$ has the following expression:

$$
\begin{aligned}
\left|\mathcal{R}_{i, f_{\mathrm{MO}}}^{+, \mathrm{CDW}-\mathrm{EII}}\left(\vec{\eta}, \Omega_{b}\right)\right|^{2}= & \sum_{h}\left|\mathcal{R}_{h_{\mathrm{MO}}}^{+, \text {eff }}\left(\vec{\eta}, \Omega_{b}\right)\right|^{2}+\cos [(\vec{k}+\vec{K}) \cdot \vec{b}]\left[\mathcal{R}_{1_{\mathrm{MO}}}^{+ \text {eff* }}\left(\vec{\eta}, \Omega_{b}\right) \mathcal{R}_{2_{\mathrm{MO}}}^{+, \text {eff }}\left(\vec{\eta}, \Omega_{b}\right)+\mathcal{R}_{1_{\mathrm{MO}}}^{+, \text {eff }}\left(\vec{\eta}, \Omega_{b}\right) \mathcal{R}_{2 \mathrm{MO}}^{+, \text {eff* }}\left(\vec{\eta}, \Omega_{b}\right)\right] \\
& -2 i \sin [(\vec{k}+\vec{K}) \cdot \vec{b}]\left[\mathcal{R}_{1_{\mathrm{MO}}^{+, \text {eff* }}}\left(\vec{\eta}, \Omega_{b}\right) \mathcal{R}_{2_{\mathrm{MO}}}^{+ \text {eff }}\left(\vec{\eta}, \Omega_{b}\right)-\mathcal{R}_{1_{\mathrm{MO}}}^{+ \text {eff }}\left(\vec{\eta}, \Omega_{b}\right) \mathcal{R}_{2_{\mathrm{MO}}}^{+, \text {eff* }}\left(\vec{\eta}, \Omega_{b}\right)\right] \\
= & S_{\mathrm{MO}, d}\left(\vec{\eta}, \Omega_{b}\right)+S_{\mathrm{MO}, i_{1}}\left(\vec{\eta}, \Omega_{b}\right)+S_{\mathrm{MO}, i_{2}}\left(\vec{\eta}, \Omega_{b}\right),
\end{aligned}
$$

where $\vec{K}=-\vec{\eta}-K_{z} \hat{v}$ with $(-\vec{K})$ the total momentum transfer, and $\vec{b}$ is the internuclear molecular vector. The direct term $S_{\mathrm{Mo}, d}$ and the interference terms $S_{\mathrm{Mo}, i_{1}}$ and $S_{\mathrm{Mo}, i_{2}}$ correspond to the three addends defined in Eq. (4). DDCSs for the complete molecule can be obtained by adding all the partial contributions (3) corresponding to each molecular orbital of the target:

$$
\sigma^{(2)}\left(\varepsilon_{k}, \Omega_{k}\right)=\sum_{\mathrm{MO}} \sigma_{\mathrm{MO}}^{(2)}\left(\varepsilon_{k}, \Omega_{k}\right)
$$

\section{RESULTS AND DISCUSSION}

\section{A. Energy distributions of the electron DDCSs at definite emission angle}

The measured energy distribution of absolute DDCSs for electron emission is shown in the Fig. 1. The cross section decreases over several orders with increase in the electron energy. This can be attributed to the fact that at energies around a few $\mathrm{eV}$ the soft electron emission process dominates in which the electrons are emitted with very large impact parameters and hence with the maximum cross section. The sharp peak around $480 \mathrm{eV}$ corresponds to the $K-L L$ Auger electron emission [see Fig. 1(a)]. The FWHM of the Auger transition peak from different scattering angles turns out to be approximately $10 \%$ of the characteristic Auger transition energy. This is due to the fact that these are not single lines, but an admixture of multiple satellite Auger lines. These satellite lines arise due to multiple ionization of the target molecule in collisions with highly charged ions. The experimental data were compared with the theoretical calculations using the CDW-EIS model. Contributions from different $\alpha$ and $\beta$ orbitals are considered, according to the corresponding electron spin character. In general, a qualitative agreement between the theory and the experimental data is obtained for different electron emission angles, even considering the simple molecular representation used for the triplet initial molecular state. As far as the absolute agreement is concerned, one finds substantial deviation of the experimental data from theoretical values below $100 \mathrm{eV}$, specially for the forward angles [see Figs. 1(a) and 1(b)]. Moreover, it must be mentioned that the theoretical model does not take into account the Auger emission process and some devia- tions could be seen in the higher-energy region particularly at forward emission [see Fig. 1(a)]. A prominent change in shape of the experimental DDCS spectrum can be seen at $90^{\circ}$ [see Fig. 1(c)] with respect to other forward and backward angles. This change is due to the dominance of the binary encounter processes between the projectile and target electrons.

\section{B. DDCS angular distributions at definite ejection energy}

Figure 2 shows the angular distributions of the absolute electron DDCSs at different energies of the ejected secondary electrons. The solid line corresponds to the theoretical
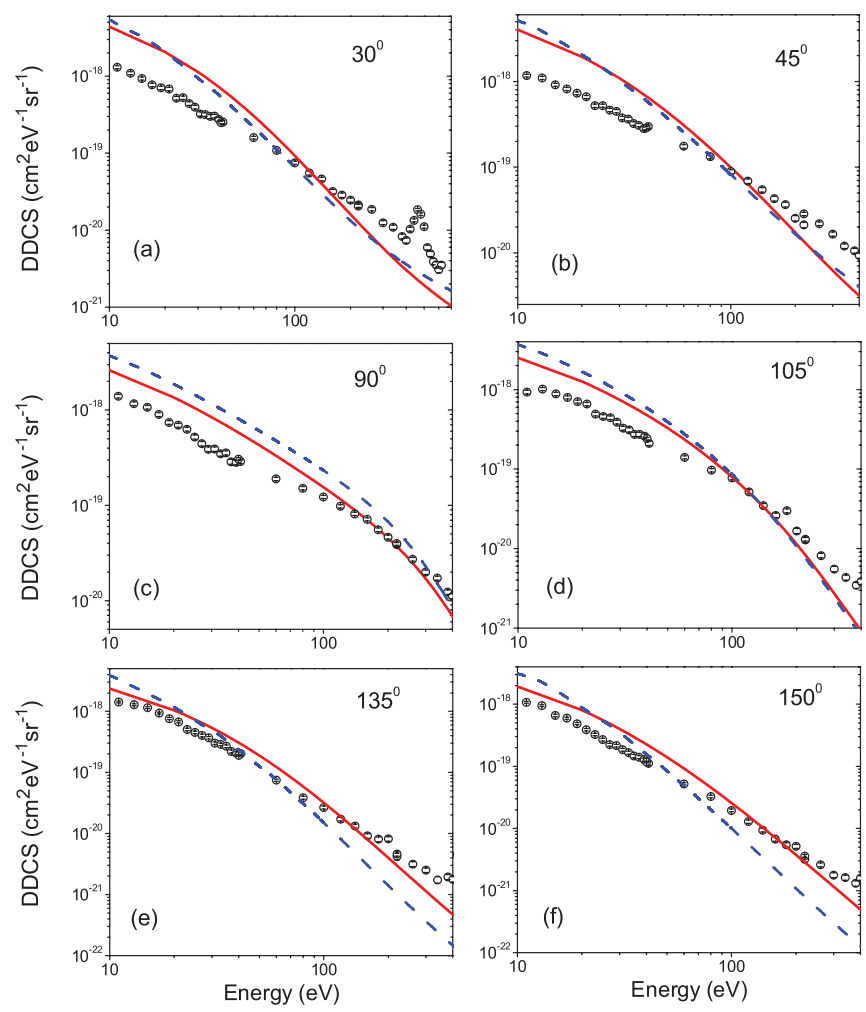

FIG. 1. (Color online) The absolute electron DDCSs for different emission angles. The solid line in each plot corresponds to the theoretical calculations using the CDW-EIS model. The dashed line in each graph corresponds to two times the atomic oxygen DDCS. 

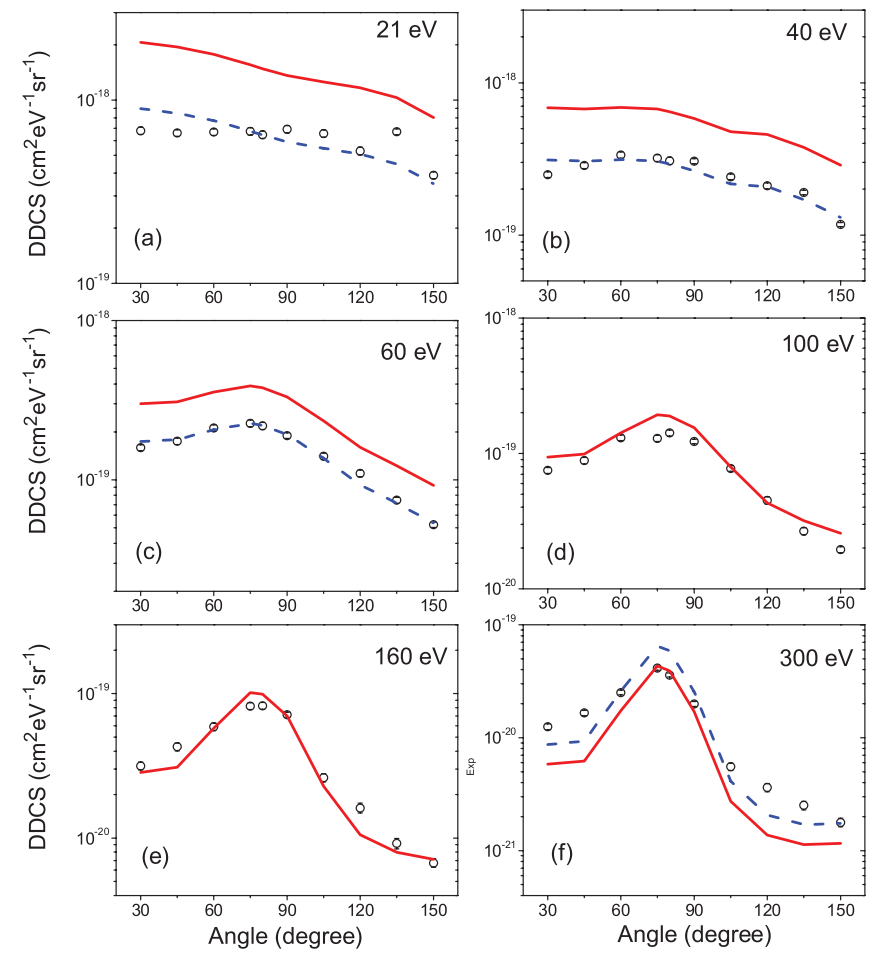

FIG. 2. (Color online) The absolute electron DDCSs at different emission energies of the secondary electrons. The solid line in each plot corresponds to the theoretical calculations using the CDW-EIS model. The dashed lines in the plots are shown to display the qualitative agreement between the theory and experiment. The "scaledown" factors in (a), (b), and (c) are 2.4, 2, and 1.4, respectively. Similarly for (f) the "scale-up" factor is 1.2.

calculations using the CDW-EIS model. At low energy, such as $21 \mathrm{eV}$ [see Fig. 2(a)], the ratio of the DDCS at $30^{\circ}$ to that at $150^{\circ}$ is about 1.74 . Similarly, at relatively high energy, such as $60 \mathrm{eV}$ [see Fig. 2(c)], the corresponding ratio is about 3.2. Now at very high energy, such as $300 \mathrm{eV}$ [see Fig. 2(f)], it is about 6.67. Clearly, the ratio between the DDCS at extreme forward angle and the DDCS at extreme backward angle increases with increase in energy of the ejected electrons. This large forward-backward angular asymmetry can be explained in terms of the two-center effects (TCE). It becomes more relevant as the ejected electron is increasingly influenced by the two Coulombic centers, namely, the receding projectile ion as well as the residual recoil ion. In this situation, the ejected electron can undergo a significant amount of successive or simultaneous deflection in the two-center field. The enhancement of the cross section in the forward angles can be attributed to the strong attraction of the emitted electrons by the receding projectiles moving in the same direction. Very low-energy electrons are assumed to be less affected by the two-center effect, because the velocities are very small compared to the velocity of the projectile ion. In the cases of 21 and $40 \mathrm{eV}$, the distributions are almost flat and no appreciable peak is observed. This is qualitatively reproduced by the CDW-EIS model although it overestimates the data. This is, however, different from the behavior observed in the cases of $\mathrm{H}_{2}$ and $\mathrm{He}$, where a peak around $75^{\circ}$ in the DDCS angular distribution was observed at emission energy as low as $15 \mathrm{eV}[7,35]$.

For higher-energy electrons the distributions gradually become more peaked around $75^{\circ}$. The difference in the shape of the distributions for low- and high-energy electrons is due to the binary nature of collisions [36]. We have noticed that the distributions are asymmetric about the peak, i.e., the cross sections at forward angles are much larger than those for backward angles, only above $40 \mathrm{eV}$ [see Fig. 2(b)]. Overall, there is a quantitative agreement between the experimental data and theoretical calculations at high ejection energies [see Fig. 2(f)]. This could be due to the fact that the CDW-EIS model gives an appropriate description of the two-center effect which is more prominent for higher ejection energies of the electrons.

\section{The angular asymmetry parameter}

The two-center effect can lead to an increase in the electron yield in the forward direction and a larger decrease in the forward direction. However, there is another governing factor, the non-Coulomb potential, that can also contribute to the forward-backward angular asymmetry. Since our target molecule $\left(\mathrm{O}_{2}\right)$ contains many electrons, the non-Coulomb potential arising from this multielectronic target should also be considered in order to explain any such asymmetry; it is anyway included in the present calculations. In order to quantify this angular asymmetry, we can introduce the angular asymmetry parameter $\alpha(k)$ as Ref. [37]

$$
\alpha(k, \theta)=\frac{\sigma^{(2)}(k, \theta)-\sigma^{(2)}(k, \pi-\theta)}{\sigma^{(2)}(k, \theta)+\sigma^{(2)}(k, \pi-\theta)},
$$

where the electron energy $\epsilon_{k}=\frac{k^{2}}{2}$ (a.u.) and $\theta$ represents extreme forward angles. By expanding $\sigma^{(2)}(k, \theta)$ in terms of the Legendre polynomials we obtain

$$
\sigma^{(2)}(k, \theta) \equiv \frac{d^{2} \sigma}{d \epsilon_{e} d \Omega_{e}}=\sum_{L} \beta_{L} P_{L}(\cos \theta),
$$

where $\beta_{L}$ is the angular anisotropy parameter and $P_{L}(\cos \theta)$ is the Legendre polynomial. As it has been shown elsewhere [37] that only values of $L$ up to 2 contribute significantly to the determination of the $\alpha$ parameter, calculating $\alpha(k)$ for $\theta=0$, and keeping only the first couple of terms in the above series, we obtain

$$
\alpha(k, \theta)=\frac{\sum_{j} \beta_{2 j+1}(k)}{\sum_{j} \beta_{2 j+1}(k)} \simeq \frac{\beta_{1}(k)}{\beta_{0}(k)+\beta_{2}(k)} .
$$

Evidently, the odd terms in the numerator correspond to the angular asymmetry while the even terms in the denominator correspond to the symmetric contributions. Since angular distributions vary slowly near 0 and $\pi$, we have used measured DDCSs at $\theta=30^{\circ}$ to approximately calculate $\alpha(k, \theta)$ as the forward-backward angular asymmetry. Figure 3 shows the velocity dependence of the angular asymmetry parameter at $\theta=30^{\circ}$. In this case, the $\alpha$ parameter varies from 0.1 to 0.8 over an energy range of 10 to $400 \mathrm{eV}$ for electrons emitted from $\mathrm{O}_{2}$. Typically the $\alpha$ parameter varies from 0.4 to 0.9 over an energy range of 1 to $100 \mathrm{eV}$ for electrons emitted from $\mathrm{He}$ in collision with fast bare carbon ions [35]. It can be seen that the asymmetry parameter increases smoothly with 


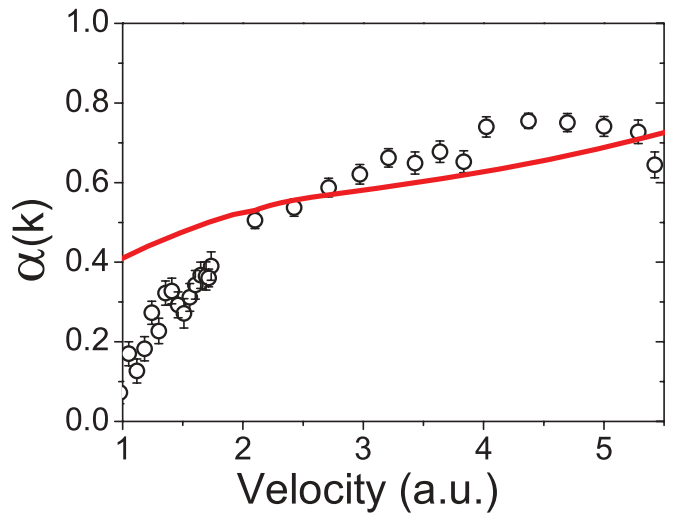

FIG. 3. (Color online) Velocity dependence of the angular asymmetry parameter.

the electron velocity for a given collision energy, a fact which can be explained in terms of the two-center effect.

\section{Single-differential cross-sections}

The DDCS spectra recorded can be used to obtain the single-differential cross sections (SDCSs) by integrating over one of the variables, either the emission angle or the ejected energy. The SDCS in angle of emission can be obtained by integrating the DDCSs of energy distributions over the ejected electron energy $\epsilon_{e}$ as

$$
\frac{d \sigma}{d \Omega_{e}}=\int_{\epsilon_{i}}^{\epsilon_{f}} \frac{d^{2} \sigma}{d \Omega_{e} d \epsilon_{e}} d \epsilon_{e} .
$$

Similarly the SDCS in ejected energy can be obtained by integrating the angular distribution of DDCSs over the solid angle of emission $\Omega_{e}$,

$$
\frac{d \sigma}{d \epsilon_{e}}=\int_{\theta_{i}}^{\theta_{f}} \frac{d^{2} \sigma}{d \Omega_{e} d \epsilon_{e}} d \Omega_{e} .
$$

Figure 4 shows the SDCSs for angular distribution of ejected electrons. The DDCS values have been integrated over an energy range of 10 to $400 \mathrm{eV}$, i.e., excluding the Auger region, for obtaining the $\operatorname{SDCS}\left(\frac{d \sigma}{d \Omega}\right)$ values. The shape of the

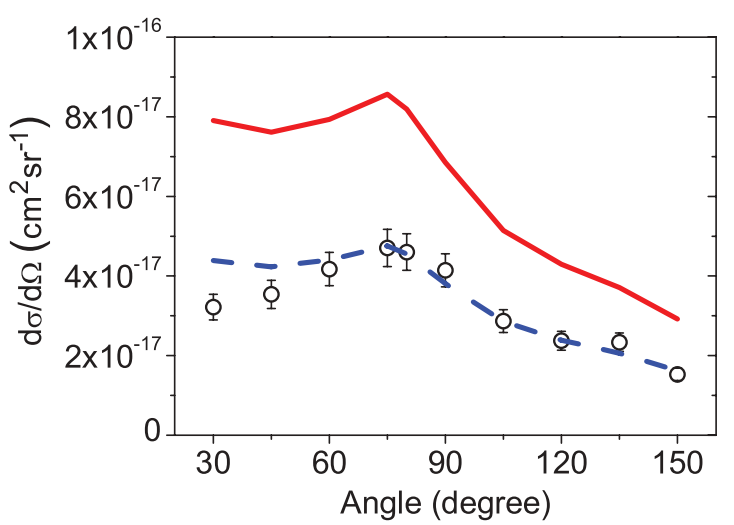

FIG. 4. (Color online) The absolute electron SDCSs at different emission angles of the secondary electrons. The solid line corresponds to the theoretical calculations using the CDW-EIS model. The dashed line represents the CDW-EIS results divided by 1.8 .

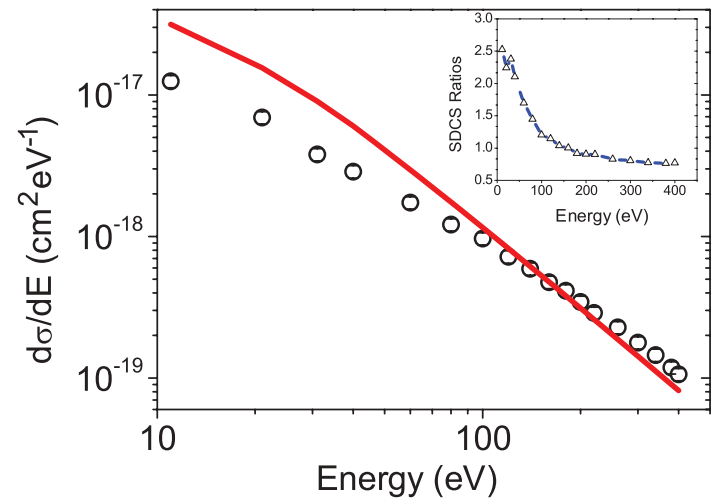

FIG. 5. (Color online) The absolute electron SDCSs at different emission energies of the secondary electrons. The solid line corresponds to the theoretical calculations using the CDW-EIS model. The inset shows the ratio theoretical calculations and experimental data. The blue line in the inset is to guide the eyes.

distribution has been well reproduced by the theoretical model. However, it overestimates the experimental data by a factor of 2.5 to 1.5 over different values of the scattering angle $\theta$. In this figure, the dashed (blue) line corresponds to the theoretical values scaled down by a factor of 1.8 , which is shown to display the qualitative agreement between experimental data and the model.

Figure 5 shows the SDCSs for the energy distribution of ejected electrons. The DDCS values have been integrated over an angular range of $\theta=30^{\circ}$ to $\theta=150^{\circ}$ for obtaining the $\operatorname{SDCS}\left(\frac{d \sigma}{d E}\right)$ values. Overall, the energy dependence of the distribution has been well reproduced by the theoretical model. However, there is a quantitative agreement between the experimental data and theoretical calculations only at energies more than $200 \mathrm{eV}$. This could be due to the large discrepancies between the theoretical model and experimental data for the DDCSs at lower ejection energies. See the inset of Fig. 5 for the ratio of $\frac{d \sigma}{d E}$ obtained from the CDW-EIS model and that obtained from experimental data. The total cross section obtained from the experimental data (interpolated from $0^{\circ}$ to $180^{\circ}$ ) is about $450 \mathrm{Mb}$, whereas the total cross section obtained from the theoretical calculations (interpolated from $0^{\circ}$ to $180^{\circ}$ ) is about $860 \mathrm{Mb}$. Evidently, the theoretical value overestimates the experimental value by an approximate factor of 1.91 .

\section{E. Interference oscillation 1. DDCS ratio}

The two-nucleon centers in $\mathrm{H}_{2}$ can be considered as two coherent sources of electrons in molecular ionization. Consequently, the electron that has reached the detector cannot be tagged a priori as from which atom it has been emitted. This phenomenon, similar to Young-type double-slit interference, results in an interference pattern for the measured electron DDCS spectra investigated as a function of the energy of emitted electrons. Angular dependency has also been observed in the frequency of the interference oscillation [38]. But the absolute DDCS varies over a few orders of magnitude over an energy range of $400 \mathrm{eV}$. Hence, the DDCS for $\mathrm{O}_{2}$ should be divided by the theoretical DDCS for atomic oxygen, in order 

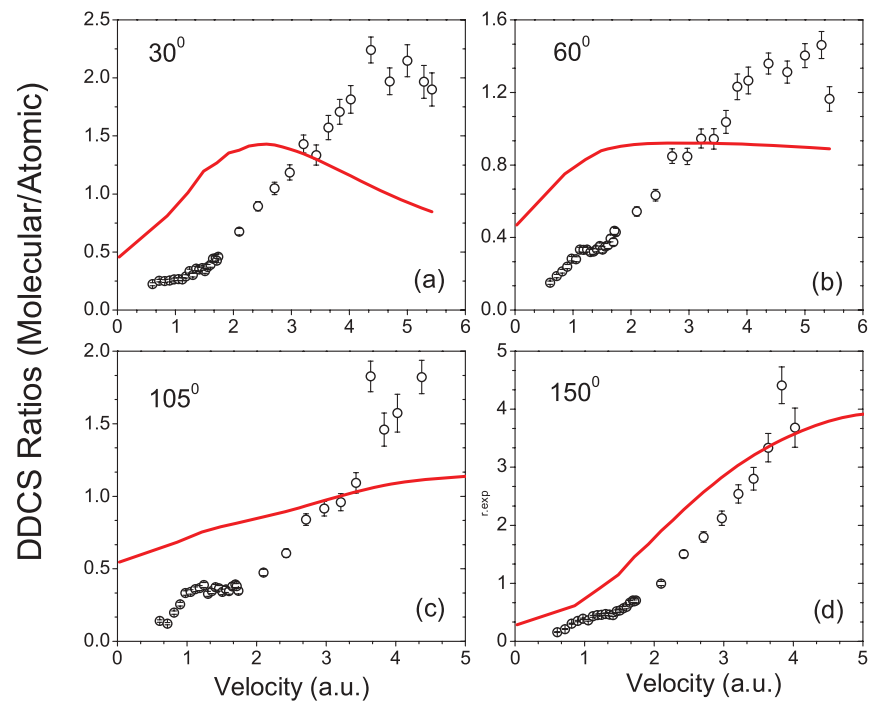

FIG. 6. (Color online) The absolute electron DDCS ratios $\sigma_{\mathrm{O} 2} / 2 \sigma_{\mathrm{O}}$ at different scattering angles the secondary electrons. The solid line in each plot corresponds to the theoretical calculations using the CDW-EIS model.

to magnify any oscillations which may be present in the DDCS for $\mathrm{O}_{2}$ but are not visible otherwise. The ratio $\sigma_{\mathrm{O}_{2}} / 2 \sigma_{\mathrm{O}}$ at $30^{\circ}$ emission angle increases monotonically from 0.5 to 4.3 a.u. of velocity and decreases thereafter with increase in velocity [see Fig. 6(a)]. As far as the absolute values are concerned there is a large disagreement between the ratio obtained from the CDW-EIS model and the ratio obtained from experimental data. If one looks at larger emission angles, such as $105^{\circ}$ [see Fig. 6(c)], both the experimental data as well as the theoretical calculations increase monotonically between 0.5 and 4.8 a.u. of velocity. Also, at extreme backward angles, such as $150^{\circ}$, the ratio $\sigma_{\mathrm{O} 2} / 2 \sigma_{\mathrm{O}}$ varies from 0.1 to a maximum of 6.0 [see Fig. 6(d)], whereas, for extreme forward angles, such as $30^{\circ}$, the ratio $\sigma_{\mathrm{O} 2} / 2 \sigma_{\mathrm{O}}$ varies from 0.3 to a maximum of 2.0 [see Fig. 6(a)]. It must be mentioned that none of the variations observed in Fig. 6, both theoretically and experimentally, show the typical oscillatory shapes of molecular double-slit interferences. Winkworth et al. [11] reported earlier on the existence of a secondary oscillation in the case of $\mathrm{O}_{2}$ under heavy-ion impact. They have compared the experimental DDCSs with "molecular" calculations using a "monocentric" description of the target orbitals. Thus they obtain the ratio between "normalized" experimental and theoretical results. It should be noted that they do not divide their experiments by twice the atomic oxygen DDCS as we have done in the present work. Then the obtained ratio was divided further by a linear fitting to eliminate the underestimation of the theoretical compared to the experimental DDCSs at high electron energies. These authors found oscillations which they claimed not as any primary interferences but as secondary ones due to intramolecular electron scattering in both centers of the target. It was not explained how and why only the second-order oscillations arise but not the first order (which should have been stronger). We cannot confirm the presence of similar oscillations. It is worth noting that we have also divided the DDCS ratios $\sigma_{\mathrm{O} 2} / 2 \sigma_{\mathrm{O}}$ by the best-fitted straight

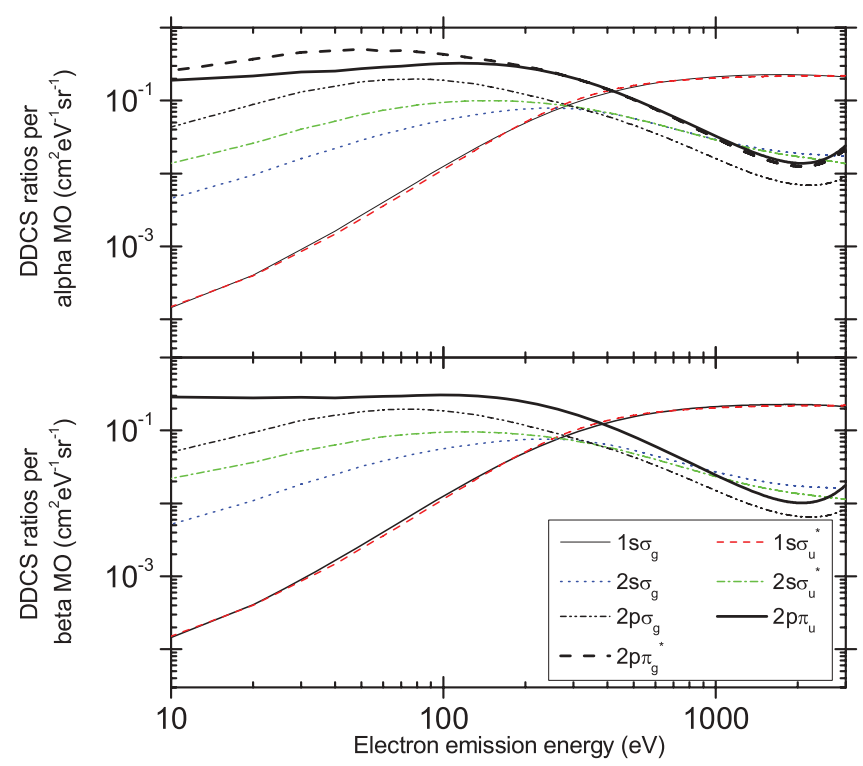

FIG. 7. (Color online) The DDCS ratio contributions from the different orbitals at $30^{\circ}$ angular emission.

line for each angle. In these results also (not shown) we cannot identify any variations indicating an oscillatory dependence.

In order to understand this behavior we present in Fig. 7 the contributions to the DDCS ratios from each $\alpha$ and $\beta$ molecular orbital for an ejection angle of $30^{\circ}$. In contrast to the $\mathrm{N}_{2}$ case (see [28]), for $\mathrm{O}_{2}$ the oscillations are much more smooth and regular, showing that all external orbitals are almost in phase while the inner ones are clearly shifted with the former. When all these contributions are added the curve shown in Fig. 6 is obtained.

\section{Asymmetry parameter}

The $\alpha$ parameter (Fig. 3, discussed earlier) does not show any oscillation as a function of the electron velocity. However, this also is in contrast to the earlier result for $\mathrm{H}_{2}$, wherein a prominent oscillatory behavior was observed in the the velocity dependence of the $\alpha$ parameter [7]. This particular phenomenon in the case of $\mathrm{H}_{2}$ is well understood in terms of the double-slit interference in the case of diatomic molecules. Although oxygen is a diatomic molecule, the reason for the absence of any oscillatory behavior in the $\alpha$ parameter can be attributed to the several molecular orbitals present in $\mathrm{O}_{2}$ for which the contribution from each MO may be phase shifted so that, when added, all them tend to cancel each other, resulting in no oscillation. However, we should note that a weak oscillation is still preserved in the theoretical calculations. The nonexistence of oscillation in $\alpha(k)$ rules out the possibility of any primary oscillation. It is not possible to use this observable for determining the presence or absence of any secondary oscillation. However, no such oscillation is found in the data as discussed in context of the Fig. 6.

\section{CONCLUSION}

In conclusion we have reported the doubly differential cross section as a function of electron energy and emission 
angle for a multielectronic system, the oxygen molecule. The DDCS values obtained from the experiment agree, in general, qualitatively with those from the CDW-EIS calculations. However, a quantitative agreement has been found in some of the $\theta-E$ windows involving relatively high energies (around 100 to $200 \mathrm{eV}$ ) of the emitted electrons. The forward-backward angular asymmetry parameter has been deduced from the DDCS values at $30^{\circ}$ and $150^{\circ}$. It increases monotonically with increase in the velocity of the ejected electrons. No oscillatory structure in the $\alpha$ parameter has been seen. The single-differential cross sections obtained by integrating the experimental DDCSs are in qualitative agreement with the theoretical calculations. However, the absolute value of the total cross section is overestimated by the CDW-EIS calculations used. No prominent oscillatory behavior, either in the experimental data or in the theoretical calculations, has been observed in the DDCS ratio $\sigma_{\mathrm{O} 2} / 2 \sigma_{\mathrm{O}}$. No primary or secondary oscillations have been observed, in contrast to the conclusions in Ref. [11]. A theoretical explanation for this behavior has been provided in terms of the molecular orbital picture. The present work further supports the point of view developed in the theoretical formalism [28].

\section{ACKNOWLEDGMENTS}

We would like to thank the staff at BARC-TIFR Pelletron accelerator facility for smooth operation of the machine. One of the authors (F.M.) acknowledges the financial support from the MICINN Projects No. FIS2010-15127 and No. CSD 200700010. C.A.T., R.D.R., and F.M. acknowledge the Programa de Cooperación Interuniversitaria e Investigación Científica entre España e Iberoamérica AECID Project No. A2/039631/11.
[1] D. S. F. Crothers and J. F. McCann, J. Phys. B 16, 3229 (1983).

[2] P. D. Fainstein, V. H. Ponce, and R. D. Rivarola, J. Phys. B 24, 3091 (1991).

[3] N. Stolterfoht, H. Platten, G. Schiwietz, D. Schneider, L. Gulyas, P. D. Fainstein, and A. Salin, Phys. Rev. A 52, 3796 (1995).

[4] G. W. Kerby III, M. W. Gealy, Y.-Y. Hsu, M. E. Rudd, D. R. Schultz, and C. O. Reinhold, Phys. Rev. A 51, 2256 (1995).

[5] L. C. Tribedi, P. Richard, Y. D. Wang, C. D. Lin, and R. E. Olson, Phys. Rev. Lett. 77, 3767 (1996).

[6] L. C. Tribedi, P. Richard, L. Gulyas, and M. E. Rudd, Phys. Rev. A 63, 062724 (2001).

[7] D. Misra, A. Kelkar, U. Kadhane, A. Kumar, Y. P. Singh, L. C. Tribedi, and P. D. Fainstein, Phys. Rev. A 75, 052712 (2007).

[8] R. D. DuBois and M. E. Rudd, Phys. Rev. A 17, 843 (1978).

[9] S. Suárez, C. Garibotti, G. Bernardi, P. Focke, and W. Meckbach, Phys. Rev. A 48, 4339 (1993).

[10] J. L. Baran, S. Das, F. Járái-Szábo, K. Póra, L. Nagy, and J. A. Tanis, Phys. Rev. A 78, 012710 (2008).

[11] M. Winkworth, P. D. Fainstein, M. E. Galassi, J. L. Baran, B. S. Dassanayake, S. Das, A. Kayani, and J. A. Tanis, Nucl. Instrum. Methods Phys. Res., Sect. B 267, 373 (2009).

[12] H. D. Cohen and U. Fano, Phys. Rev. 150, 30 (1966).

[13] D. K. Jain and S. P. Khare, Phys. Lett. A 63, 237 (1977).

[14] A. Jain, A. N. Tripathi, and M. K. Srivastava, Phys. Rev. A 20, 2352 (1979).

[15] N. Stolterfoht, B. Sulik, V. Hoffmann, B. Skogvall, J. Y. Chesnel, J. Rangama, F. Fremont, D. Hennecart, A. Cassimi, X. Husson, A. L. Landers, J. A. Tanis, M. E. Galassi, and R. D. Rivarola, Phys. Rev. Lett. 87, 023201 (2001).

[16] D. Misra, U. Kadhane, Y. P. Singh, L. C. Tribedi, P. D. Fainstein, and P. Richard, Phys. Rev. Lett. 92, 153201 (2004).

[17] J. A. Tanis, S. Hossain, B. Sulik, and N. Stolterfoht, Phys. Rev. Lett. 95, 079301 (2005).

[18] D. Misra, U. Kadhane, Y. P. Singh, L. C. Tribedi, P. D. Fainstein, and P. Richard, Phys. Rev. Lett. 95, 079302 (2005).

[19] F. Frémont, A. Hajaji, A. Naja, C. Leclercq, J. Soret, J. A. Tanis, B. Sulik, and J.-Y. Chesnel, Phys. Rev. A 72, 050704(R) (2005).

[20] D. Misra, A. Kelkar, U. Kadhane, A. Kumar, L. C. Tribedi, and P. D. Fainstein, Phys. Rev. A 74, 060701(R) (2006).
[21] J. A. Tanis, J.-Y. Chesnel, B. Sulik, B. Skogvall, P. Sobocinski, A. Cassimi, J.-P. Grandin, L. Adoui, D. Hennecart, and N. Stolterfoht, Phys. Rev. A 74, 022707 (2006).

[22] L. Ph. H. Schmidt, S. Schössler, F. Afaneh, M. Schöffler, K. E. Stiebing, H. Schmidt-Böcking, and R. Dörner, Phys. Rev. Lett. 101, 173202 (2008).

[23] M. E. Galassi, R. D. Rivarola, P. D. Fainstein, and N. Stolterfoht, Phys. Rev. A 66, 052705 (2002).

[24] L. Nagy, L. Kocbach, K. Póra, and J. P. Hansen, J. Phys. B 35, L453 (2002).

[25] N. Stolterfoht, B. Sulik, B. Skogvall, J. Y. Chesnel, F. Frémont, D. Hennecart, A. Cassimi, L. Adoui, S. Hossain, and J. A. Tanis, Phys. Rev. A 69, 012701 (2004).

[26] Nicolas Sisourat, Jérémie Caillat, Alain Dubois, and P. D. Fainstein, Phys. Rev. A 76, 012718 (2007).

[27] D. Misra, A. H. Kelkar, S. Chatterjee, and L. C. Tribedi, Phys. Rev. A 80, 062701 (2009).

[28] C. A. Tachino, F. Martín, and R. D. Rivarola, J. Phys. B 45, 025201 (2011).

[29] D. Misra, K. V. Thulasiram, W. Fernandes, A. H. Kelkar, U. Kadhane, A. Kumar, Y. P. Singh, L. Gulyas, and L. C. Tribedi, Nucl. Instrum. Methods Phys. Res., Sect. B 267, 157 (2009).

[30] Channel Electron Multiplier, Dr. Sjuts Optotechnik GmbH, Germany, 2002.

[31] M. W. Gealy, G. W. Kerby III, Y.-Y. Hsu, and M. E. Rudd, Phys. Rev. A 51, 2247 (1995).

[32] M. J. Frisch et al., computer code GAUSSIAN 98, revision A11 (Gaussian Inc., Pittsburgh, PA, 1999).

[33] K. O-ohata, H. Taketa, and S. Huzinaga, J. Phys. Soc. Jpn. 21, 2306 (1966).

[34] W. J. Hehre, R. F. Stewart, and J. A. Pople, J. Chem. Phys. 51, 2657 (1969).

[35] L. C. Tribedi, P. Richard, Y. D. Wang, C. D. Lin, L. Gulyas, and M. E. Rudd, Phys. Rev. A 58, 3619 (1998).

[36] S. T. Manson, L. H. Toburen, and N. Stolterfoht, Phys. Rev. A 12, 60 (1975).

[37] P. D. Fainstein, L. Gulyás, F. Martin, and A. Salin, Phys. Rev. A 53, 3243 (1996).

[38] S. Chatterjee, D. Misra, A. Kelkar, P. D. Fainstein, and L. C. Tribedi, J. Phys. B 43, 125201 (2010). 